\title{
Pan-Cancer Clinical and Molecular Analysis of Racial Disparities
}

\author{
Olivia D. Lara, MD ID 1; Ying Wang, PhD²; Amma Asare, MD³; Tao Xu, PhD ${ }^{4}$; Hua-Sheng Chiu, PhD ; Yuexin Liu, PhD (iD 2;

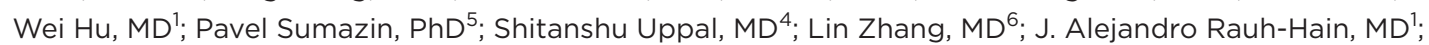 \\ and Anil K. Sood, MD ${ }^{1,7}$
}

\begin{abstract}
BACKGROUND: Racial disparities in cancer outcomes are increasingly recognized, but comprehensive analyses, including molecular studies, are limited. The objective of the current study was to perform a pan-cancer clinical and epigenetic molecular analysis of outcomes in African American (AA) and European American (EA) patients. METHODS: Cross-platform analyses using cancer databases (the Surveillance, Epidemiology, and End Results program database and the National Cancer Data Base) and a molecular database (The Cancer Genome Ancestry Atlas) were performed to evaluate clinical and epigenetic molecular differences between AA and EA patients based on genetic ancestry. RESULTS: In the primary pan-cancer survival analysis using the Surveillance, Epidemiology, and End Results database (2,045,839 patients; $87.5 \%$ EA and $12.5 \%$ AA), AA patients had higher mortality rates for 28 of 42 cancer types analyzed (hazard ratio, $>1.0$ ). AAs continued to have higher mortality in 13 cancer types after adjustment for socioeconomic variables using the National Cancer Database (5,150,023 patients; $11.6 \%$ AA and $88.4 \%$ EA). Then, molecular features of 5,283 tumors were analyzed in patients who had genetic ancestry data available (87.2\% EA and $12.8 \%$ AA). Genes were identified with altered DNA methylation along with increased microRNA expression levels unique to AA patients that are associated with cancer drug resistance. Increased miRNAs (miR-15a, miR-17, miR-130-3p, miR-181a) were noted in common among AAs with breast, kidney, thyroid, or prostate carcinomas. CONCLUSIONS: The current results identified epigenetic features in AA patients who have cancer that may contribute to higher mortality rates compared with EA patients who have cancer. Therefore, a focus on molecular signatures unique to AAs may identify actionable molecular abnormalities. Cancer 2020;126:800-807. ( 2019 American Cancer Society.
\end{abstract}

KEYWORDS: African Americans, epigenomics, health care disparities, long noncoding, microRNAs, pan-cancer, RNA.

\section{INTRODUCTION}

For many cancer types, authors have documented racial disparity in patient survival. In particular, African American (AA) patients have higher mortality rates than all other racial groups for many cancer types. ${ }^{1-3}$ For example, AA women are 40\% more likely than European American (EA) women to die of breast cancer, whereas AA men are twice as likely as EA men to die of prostate cancer. ${ }^{4}$ Many factors contribute to disparities in cancer outcomes, including socioeconomic factors, culture, diet, stress, and the macroenvironment. ${ }^{5-7}$ Research into health disparities has primarily focused on these social determinants and their impact on health. However, other observations suggest a role for molecular differences in cancer survival disparities. ${ }^{8}$ Compared with EA women, AA women have a 2-fold higher incidence of the inherently aggressive triple-negative breast cancer (TNBC). ${ }^{9}$ A comparison of TNBC in AA versus EA patients has demonstrated a gene expression signature consistent with increased loss of $B R C A 1$ expression, increased activation of insulin-like growth factor 1 receptor, and increased expression of vascular endothelial growth factor-activated genes in AA patients. ${ }^{10}$ In addition, AA patients with breast, head and neck, or endometrial cancer have higher levels of chromosomal instability and frequency of TP53 mutations and CCNE1 amplification than white patients. ${ }^{11}$

Whereas some studies have explored somatic alterations in individual cancers, ${ }^{12-14}$ little is known about how the macroenvironment affects the epigenetic landscape among the different races. ${ }^{15,16}$ Epigenetic differences are particularly relevant because they can be shaped by environmental factors, such as chronic stress, social interactions, and toxins. ${ }^{15}$ Theses epigenetic changes can function as a liaison between social, cultural, and environment factors and the genome. ${ }^{17}$ Furthermore, race is a social construct and is often a proxy for chronic stressors that minority populations face, such as low socioeconomic status,

Corresponding Author: Anil K. Sood, MD, Department of Gynecologic Oncology and Reproductive Medicine, Unit 1362, The University of Texas MD Anderson Cancer Center, 1515 Holcombe Boulevard, Houston, TX 77030 (asood@mdanderson.org).

${ }^{1}$ Department of Gynecologic Oncology and Reproductive Medicine, The University of Texas MD Anderson Cancer Center, Houston, Texas; ${ }^{2}$ Department of Bioinformatics and Computational Biology, The University of Texas MD Anderson Cancer Center, Houston, Texas; ${ }^{3}$ Baylor College of Medicine, Houston, Texas; ${ }^{4}$ Department of Gynecologic Oncology, University of Michigan, Ann Arbor, Michigan; ${ }^{5}$ Texas Children's Cancer Center, Houston, Texas; ${ }^{6}$ Department of Obstetrics and Gynecology, University of Pennsylvania, Philadelphia, Pennsylvania; ${ }^{7}$ Center for RNA Interference and Non-Coding RNA, The University of Texas MD Anderson Cancer Center, Houston, Texas

The first two authors contributed equally to this article.

We acknowledge The University of Texas MD Anderson Cancer Center Department of Scientific Publications for reviewing and editing this article.

Additional supporting information may be found in the online version of this article.

DOI: 10.1002/cncr.32598, Received: August 29, 2019; Revised: October 2, 2019; Accepted: October 7, 2019, Published online November 15, 2019 in Wiley Online Library (wileyonlinelibrary.com) 
poor access to health care, dangerous environments, and interpersonal discrimination. ${ }^{18}$ These unique stressors that a racial group experiences may affect their epigenome and ability to respond to disease, leading to poor outcome and response to therapy.

In this study, we used data from 2 large cancer registries to perform a pan-cancer mortality analysis of AAs compared with EAs. We also evaluated differences in epigenetic modifications, including noncoding RNA (microRNA [miRNA] and long-noncoding RNA [lncRNA]) expression and DNA methylation, in the 2 groups. We believe epigenetic modifications unique to AAs may contribute to differences in clinical outcomes.

\section{MATERIALS AND METHODS}

\section{Data Sources}

In our primary survival analysis, we used data from the Surveillance, Epidemiology, and End Results (SEER) program database (version 9), which contains information on cancer incidence and survival from 17 population-based cancer registries covering approximately $28 \%$ of the US population. Because the SEER database lacks comprehensive sociodemographic information, a secondary survival analysis using data from the National Cancer Database (NCDB), another US cancer registry, was performed. The NCDB is a nationwide oncology outcomes database that includes information on approximately $70 \%$ of all invasive incident cancers diagnosed in the United States. Tumor registrars at participating hospitals document patient, tumor, and treatment characteristics (including information about initial surgery, chemotherapy, and radiotherapy) in addition to survival. The NCDB population consists of patients who received cancer care (treatment or diagnosis) from among 1400 cancer programs accredited by the Commission on Cancer. These survival data were paired with molecular data from The Cancer Genome Atlas (TCGA). ${ }^{19}$ To define the AA and EA patient groups, data from the Cancer Genome Ancestry Atlas (TCGAA) ${ }^{11}$ were used. TCGA contains data such as clinical information, histopathology, and molecular information derived from information on samples obtained from more than 11,000 patients. TCGA miRNA and lncRNA sequencing data used in the current study were analyzed on the Illumina HiSeq platform, whereas TCGA DNA methylation data were analyzed on the Illumina HM450k platform (Illumina, Inc).

\section{Study Population, Covariates, and Statistical Analyses}

All cancer cases documented from 2000 to 2015 in the SEER 9 registry research data were identified. Only patients classified as EA or AA were included in our study. In addition, patients who had a prior history of cancer with a survival or follow-up time shorter than 1 month, who were aged $>100$ years at diagnosis, who had noncancer causes of death, who had tumor types found in fewer than 200 cases in the registry, or who had missing age or survival data were excluded. The patients had a total of 42 primary tumor types. The primary outcome was overall survival in AA and EA patients (see Supporting Information).

For cancer types identified in the SEER survival analyses with hazard ratios (HRs) for mortality $>1.0$ in AAs compared with EAs, a secondary survival analysis was performed for cancer types in the NCDB. The cohort is defined in the online Supporting Information. Only tumor types with available TCGA data on molecular correlations were included in this analysis (see Supporting Methods and Supporting Fig. 1).

Patients with 13 primary tumor types that had a persistent survival disadvantage according to the NCDB survival analysis were subjected to molecular analyses. On the basis of the genetic ancestry information from TCGAA, EA and AA patients in TCGA were included in our study. This differed from SEER and NCDB, which rely on self-identified race to identify AA and EA groups. The number of AA patients with skin cutaneous melanoma in TCGA was insufficient for testing, so this group was excluded from all analyses. The distribution of EA and AA patients in TCGA according to tumor type is shown in Supporting Table 1. For each TCGA tumor type of interest, $U$ tests were used to compare DNA methylation profiles, and feature-by-feature $t$ tests were run to compare the miRNA sequencing profiles for the $\mathrm{AA}$ and EA groups. A $\beta$-uniform mixture model $^{20}$ was used to adjust for multiple comparisons and estimate significant features of AA race at different false-discovery rates (FDRs). LncRNA expression profiles were normalized using The Atlas of Noncoding RNA in Cancer (https://bioinformatics.mdanderson.org/public-software/ tanric/) $)^{21,22}$ based on TCGA RNA sequencing data in 11 tumor data sets, with estimates of expression levels represented in reads per kilobase million. The significance of the differential expression of each lncRNA, comparing its expression estimates between AA and EA patients with cancer, was computed using $U$ tests while controlling for the FDR for each tumor type. For invasive breast (BRCA) and uterine corpus endometrial (UCEC) carcinomas, the analyses were performed independently for TNBC and non-TNBC cases and for endometrioid endometrial adenocarcinoma (EEA) and non-EEA cases, respectively. 
To avoid low signal-to-noise instances, only lncRNAs with mean absolute deviation scores $>0.1$ were included in the final analysis.

To identify common biologic processes that potentially explain the association between AA race and overall survival, pathway analyses were performed using Ingenuity Pathway Analysis software (version 46901286; Ingenuity Systems). After comparing DNA methylation profiles of EA and AA patients for each TCGA tumor type, probes with the most extreme $P$ values were extracted and mapped to human genes. Probes with $\beta$ differences $>0.1$ at an FDR of 0.1 were considered to represent significant changes in methylation. For a gene to which multiple probes were mapped, the probe with the lowest $P$ value was selected to represent that gene. After comparing miRNA expression profiles for each tumor type, only miRNAs that were significant at a $P<.05$ were mapped. Additional pathway analysis of differentially methylated genes was performed using gene set enrichment analysis software and the Molecular Signature Database (Broad Institute and the University of California, San Diego). ${ }^{23}$ Depicted pathways have FDR $<.05$ and an absolute normalized enrichment score $>1.5$. Unsupervised hierarchical clustering, heatmap rendering, and figure creation were performed in the R programming language (R Foundation for Statistical Computing).

\section{RESULTS}

\section{Survival Analysis}

We first focused on overall survival differences between AA and EA patients using the SEER data. After applying the exclusion criteria, 2,045,839 patients who had complete data (12.5\% AA and $87.5 \%$ EA) were available for analysis. We sorted them into 42 different cancer type populations, ranging from 361,847 patients with breast carcinoma to 188 with placental cancer. Supporting Table 2 displays the clinical characteristics of the SEER study patients. Among all patients, AA race was associated with an increased risk of death for a majority of the cancer types (see Supporting Fig. 2). Specifically, 28 cancer types were associated with an increased risk of death, with HRs $>1.0$. To determine whether the survival outcome was related to sociodemographic factors, we performed a secondary survival analysis using the NCDB data. We focused on 19 cancer types associated with a survival disadvantage that had TCGA data available for molecular analyses. The NCDB data were available on a total of 5,150,023 patients (11.6\% AA and $88.4 \%$ EA). Supporting Table 3 in displays the clinical characteristics of the NCDB study patients. In the NCDB and SEER survival analyses, we observed persistently higher mortality for 13 tumor types in AA patients (HR, >1.0) compared with 6 tumor types in which the higher mortality rate was lowered after adjustment for socioeconomic variables (Fig. 1). We then performed molecular analyses for cancer types with increased mortality identified in both the NCDB and SEER data sets using TCGA data.

\section{MiRNA Analysis}

We analyzed genetic ancestry data for 5283 tumors across 12 tumor types, including $12.8 \%$ obtained from AAs and $87.2 \%$ obtained from EAs. First, we compared miRNA expression levels in AA and EA patients. Of the 12 tumor types analyzed, we focused on the 5 types with the highest numbers of significantly differentially expressed miRNAs at an FDR of 0.1: BRCA (300 miRNAs), kidney renal clear cell carcinoma (KIRC) (408 miRNAs), prostate adenocarcinoma (PRAD) (102 miRNAs), thyroid carcinoma (THCA) (63 miRNAs), and UCEC (177 miRNAs). All differentially expressed miRNAs are listed according to tumor type in Supporting Table 4. Unsupervised clustering analysis of the most variable miRNAs in AAs revealed similarities between KIRC, PRAD, THCA, and BRCA tumors (Fig. 2A) We performed pathway analysis to determine the clinical significance and biologic functions of the differentially expressed miRNAs for each cancer type. For all cancer types, differentially expressed miRNAs were associated with cancer drug resistance pathways through cancer drug efflux (see Supporting Fig. 3). Also, for all cancer types, 11 miRNAs were commonly dysregulated in AAs (Table 1). ${ }^{24-37}$ Investigation of these miRNAs within published data indicates their role in oncogenesis. Notably, the expression of miRNAs miR15a, miR-17, and miR-130-3p was frequently elevated in patients with BRCA, KIRC, prostate carcinoma, and THCA.

\section{LncRNA Analysis}

To characterize dysregulation of lncRNA expression that may be associated with AA race, we analyzed lncRNA expression in patients with 11 cancer types who had adequate sample sizes. The dysregulated lncRNAs by cancer type are listed in Supporting Table 5. Of these 11 cancer types, only 4 tumor types had $\geq 20$ dysregulated lncRNAs associated with AA race: THCA (20 lncRNAs), PRAD (80 lncRNAs), BRCA (77 lncRNAs), and KIRC (34 lncRNAs) (see Supporting Fig. 4). Greater than $70 \%$ of the lncRNAs were unique to specific cancer types. 


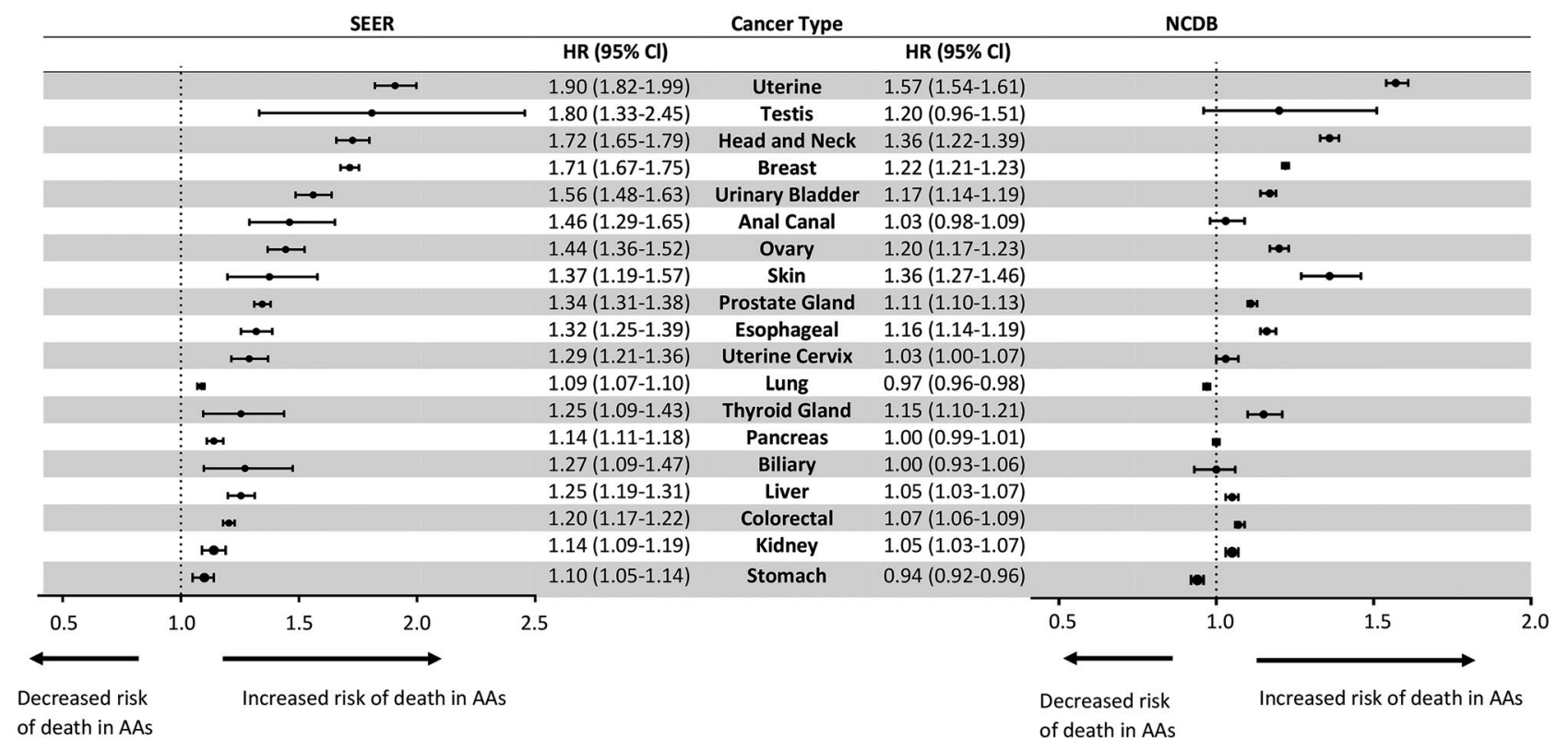

Figure 1. The risk of death in African American (AA) patients with cancer is illustrated according to tumor type. Shown are results of multivariate analysis of the risk of death in AA patients with cancer in the Surveillance, Epidemiology, and End Results (SEER) and National Cancer Data Base (NCDB) databases. HR indicates hazard ratio.

\section{DNA Methylation}

Next, we analyzed DNA methylation and gene expression data for all cancer types. We identified 9 cancer types associated with dysregulated gene methylation in AA race: bladder urothelial carcinoma (30 genes), BRCA (325 genes), colon adenocarcinoma (294 genes), esophageal carcinoma (541 genes), head and neck carcinoma (204 genes), KIRC (263 genes), PRAD (269 genes), THCA (482 genes), and UCEC (157 genes) (see Supporting Table 6). Unsupervised cluster analysis of DNA methylation and gene expression was performed on cancer types that had $>100$ genes affected (Fig. 2B). Among 7 of 8 cancer types, we identified hypomethylation of TRPC5 (transient receptor potential channel C5), S100A14 (S100 calcium binding protein A14), and MIR662 in AA tumors compared with EA tumors, leading to increased gene expression. These alterations are known to elicit resistance to chemotherapy ${ }^{38-41}$ and constitute potential therapeutic targets. In addition, we observed that esophageal carcinoma tumors presented distinct sets of DNA methylation compared with other cancer types, with 466 of 541 unique genes (86\%) affected. This may represent the already known effect of environmental exposure on molecular alterations of esophageal carcinogenesis. ${ }^{42}$ Finally, the gene set enrichment analysis of biological pathways enriched in genes with altered DNA methylation in AAs across 7 cancer types revealed an enrichment in metabolism, development, and signaling pathways (see Supporting Fig. 5).

\section{Tumor Subtyping}

Finally, we compared histologic subtypes in AA patients with BRCA and UCEC. In BRCA, TNBC is an aggressive tumor phenotype more frequently diagnosed in $A A$ women than in EA women. ${ }^{43}$ Similarly, AA women are more likely than EA women to be diagnosed with type 2 (non-EEA) uterine tumors, which are typically more aggressive than other uterine tumor subtypes. ${ }^{44}$ Therefore, we compared epigenetic modifications in TNBC versus non-TNBC cases and EEA versus non-EEA cases in AA and EA patients. AA TNBC and non-TNBC cases were associated with 274 and 41 differentially expressed miRNAs, respectively. We performed pathway analysis of individual data sets (TNBC and non-TNBC) and then compared the predicted activation of functional pathways using Ingenuity Pathway Analysis software. AA miRNA expression data on TNBC were associated with an increase in the angiogenesis pathway, whereas miRNA expression data from AAs with non-TNBC tumor types were associated with a decrease in the angiogenesis pathway (Table 2). In addition, all UCECs with dysregulated miRNA expression were of the EEA subtype; therefore, 
A

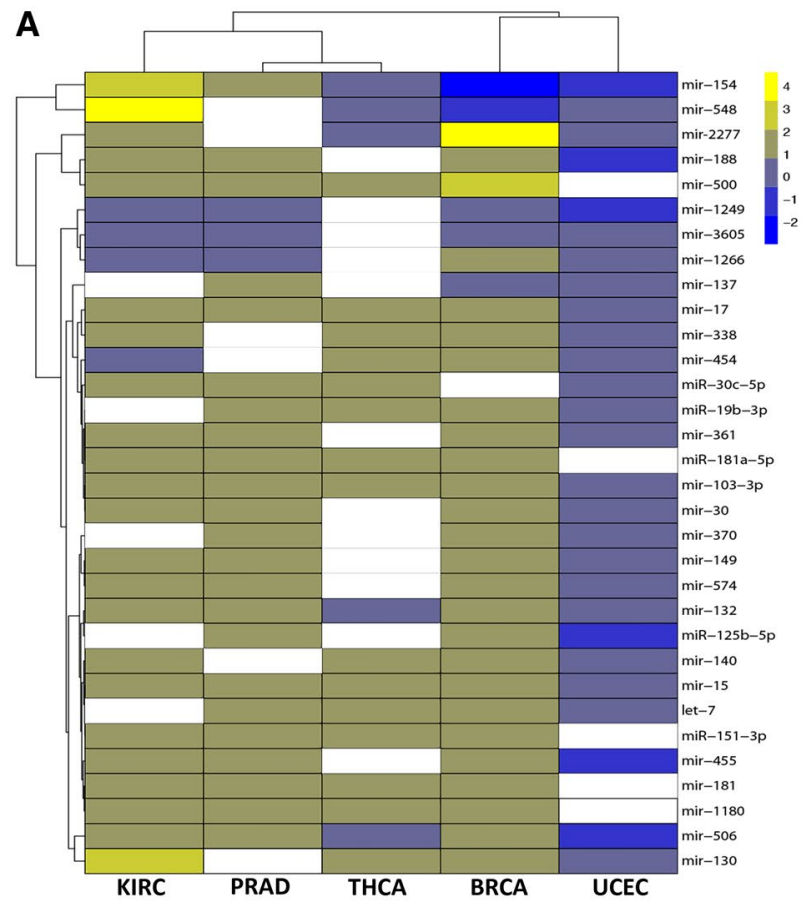

B

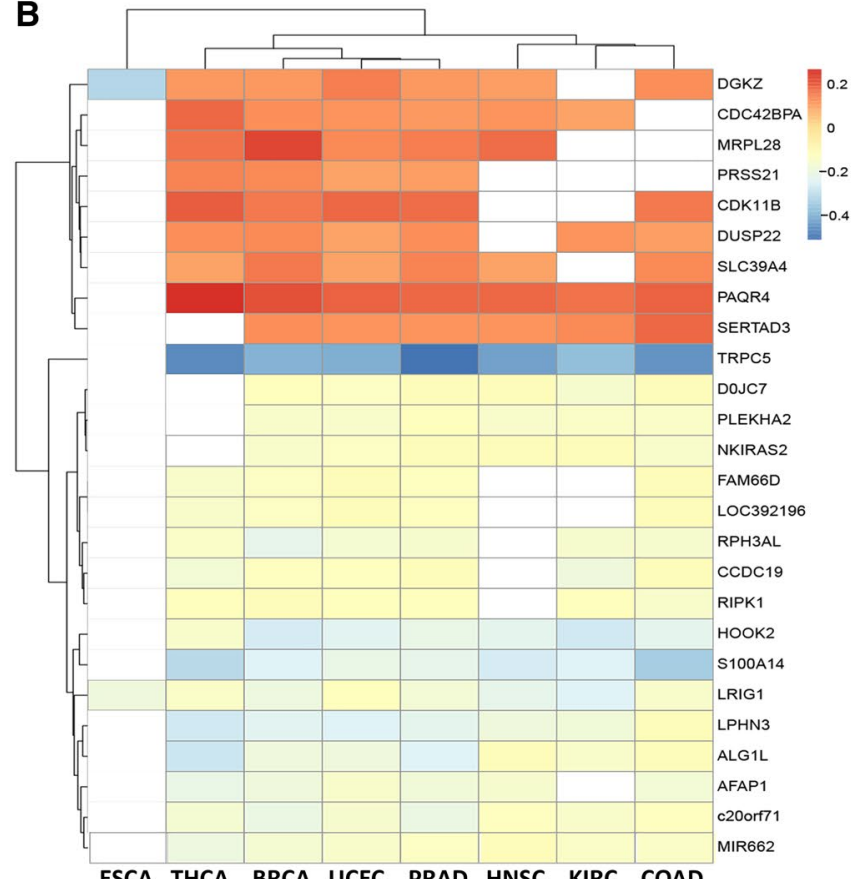

Figure 2. Features of African American (AA) patients in The Cancer Genome Atlas are illustrated. Unsupervised hierarchical clustering and heatmaps of (A) the top commonly dysregulated microRNA and (B) DNA methylation targets in AA versus European American (EA) patients with cancer from 5283 tumors. (A) Fold changes in AA vs EA patients are illustrated for significant common microRNAs in 5 cancer types. (B) $\Delta \beta$ values are illustrated in AA versus EA patients for top loci. Red boxes indicate relative hypermethylation in AA patients, blue boxes note relative hypomethylation in AA patients, and white boxes represent genes with no significant alteration in AA versus EA patients. BRCA, indicates breast cancer; COAD, colon adenocarcinoma; ESCA, esophageal carcinoma; HNSC, head and neck squamous cell carcinoma; KIRC, kidney renal clear cell carcinoma; PRAD, prostate adenocarcinoma; THCA, thyroid carcinoma; UCEC, uterine corpus endometrial carcinoma.

TABLE 1. MicroRNAs Commonly Dysregulated in African American Patients With Cancer

\begin{tabular}{|c|c|c|c|c|c|c|c|}
\hline \multirow{2}{*}{$\begin{array}{l}\text { MicroRNA } \\
\text { Component }\end{array}$} & \multicolumn{5}{|c|}{ Expression Level in AAs } & \multirow[b]{2}{*}{ Characteristic } & \multirow[b]{2}{*}{ Reference(s) } \\
\hline & UCEC & THCA & PRAD & BRCA & $\mathrm{KIRC}$ & & \\
\hline miR-27a & Low & - & - & High & High & $\begin{array}{l}\text { Resistance to } 5 \text {-fluorouracil mediated by } \\
\mathrm{miR}-27 \mathrm{a} / \mathrm{b}\end{array}$ & Geretto $2017^{24}$ \\
\hline$m i R-331-5 p$ & Low & - & - & - & - & - & \\
\hline$m i R-130 a$ & High & High & - & High & High & $\begin{array}{l}\text { Potential diagnostic marker for breast, } \\
\text { gastric, ovarian carcinomas }\end{array}$ & $\begin{array}{l}\text { Jiang } 2015,{ }^{25} \text { Zhang } 2017,{ }^{26} \\
\text { Sueta } 2017^{27}\end{array}$ \\
\hline miR-328 & Low & - & - & - & - & $\begin{array}{l}\text { Overexpression can improve radiosensitivity } \\
\text { of NSCLC }\end{array}$ & Ma $2016^{28}$ \\
\hline miR-181a & - & High & High & High & High & $\begin{array}{l}\text { Regulatory role in NSCLC pancreatic, } \\
\text { gastric, and colon carcinomas }\end{array}$ & $\begin{array}{l}\text { Feng } 2018,{ }^{29} \text { Shi } 2017,^{30} \\
\text { Meijer } 2018^{31}\end{array}$ \\
\hline $\operatorname{miR}-133 a$ & - & - & - & - & High & $\begin{array}{l}\text { Associated with aggressive bladder } \\
\text { carcinoma }\end{array}$ & Pignot $2013^{32}$ \\
\hline miR-379 & - & - & - & High & High & - & \\
\hline miR-154 & Low & - & High & Low & High & $\begin{array}{l}\text { Prognostic significance for renal cell } \\
\text { carcinoma }\end{array}$ & $\operatorname{Lin} 2018^{33}$ \\
\hline miR-15a & High & High & High & High & High & $\begin{array}{l}\text { miRNAs encoded by miR-15/16 may } \\
\text { function as tumor suppressors }\end{array}$ & Aqeilan $2009^{34}$ \\
\hline miR-17 & High & High & High & High & High & Overexpressed in various solid tumors & Zhu $2018^{35}$ \\
\hline miR-103-3p & High & High & High & High & High & $\begin{array}{l}\text { Considered an onco-miR in gastric, and } \\
\text { bladder carcinomas }\end{array}$ & Egawa $2016,{ }^{36}$ Duan $2016^{37}$ \\
\hline
\end{tabular}

Abbreviations: AA, African American; BRCA, breast cancer; KIRC, kidney renal clear cell carcinoma; miR, microRNA; NSCLC, non-small-cell lung cancer; PRAD, prostate adenocarcinoma; THCA, thyroid carcinoma; UCEC, uterine corpus endometrial carcinoma. 
TABLE 2. Disease and Functional Networks in African American Patients With Triple-Negative Breast Cancer and Non-Triple-Negative Breast Cancer Based on MicroRNA Expression

\begin{tabular}{|c|c|c|c|c|c|}
\hline Tumor Type & $\begin{array}{l}\text { Diseases or Functions } \\
\text { Annotation }\end{array}$ & $P$ & $\begin{array}{l}\text { Predicted } \\
\text { Activation }\end{array}$ & Z Score & Associated MicroRNAs \\
\hline TNBC & Angiogenesis & $1.64 \times 10^{-3}$ & Increased & 0.748 & mir-21, mir-27, mir-29, mir-361 \\
\hline Non-TNBC & Angiogenesis & $2.99 \times 10^{-9}$ & Decreased & -1.890 & $\begin{array}{l}\text { mir-135, mir-17, mir-154, mir-320, mir-19, mir-130, } \\
\text { mir-181, mir-185, mir-1301, mir-217, mir-181a-5p, } \\
\text { mir-15, mir-221, mir-361, mir-8, mir-103, mir-132, } \\
\text { mir-146, let-7, mir-25, mir-30, mir-21, mir-320b }\end{array}$ \\
\hline
\end{tabular}

Abbreviations: mir, microRNA; non-TNBC, non-triple-negative breast cancer; TNBC, triple-negative breast cancer.

we were not able to compare miRNA expression profiles in the EEA versus non-EEA cases. TRPC5, the most significantly hypomethylated gene in the BRCA and UCEC data sets, was overexpressed in all histological subtypes. We found significant differences in IncRNA expression associated with AA race only in the non-TNBC and EEA groups (see Supporting Table 5).

\section{DISCUSSION}

Our findings demonstrate that AAs have a higher risk of death for many cancer types compared with EAs. This greater risk of death was consistent across 2 large databases of patients with cancer and persisted despite controlling for socioeconomic factors, access to care, and insurance status. Furthermore, we observed that, among these cancer types with a persistent survival disadvantage in AAs, epigenetic modifications unique to AAs may have influenced tumor biology and response to therapy.

This knowledge adds to a growing body of evidence that racial disparities may be caused by genetic and biological differences. ${ }^{45}$ For instance, differential expression of inflammatory mediators, such as IL-6, and inflammatory cytokines have been found to be disproportionally increased in AA patients with breast cancer. ${ }^{46}$ In addition, both mediators of angiogenesis (vascular endothelial growth factor), and immune cells with a tumor-promoting phenotype (tumor-associated macrophages) have been found to be increased in tumors from AA patients. ${ }^{47}$ The genetic basis for these tumor-promoting events has yet to be elucidated but suggests inherent racial differences.

The strengths of this study are the use of 2 large, diverse, nationally representative samples of patients paired with an equally diverse molecular database. We adjusted for several measures of socioeconomic status that could confound the relationship between race and survival. Despite controlling for these variables, we found a persistent cancer survival disadvantage in AA patients. Furthermore, using TCGAA, we were able to reliably assign tumor types to specific races with certainty based on integrated computational algorithms to conclude the genetic ancestry of TCGA patients at global and local levels. Previous studies using TCGA data relied on self-identified race or ethnicity, which were challenging and limited the ability to elucidate the genetic contribution to cancer disparities. ${ }^{48}$ In addition, despite emerging interests in the noncoding genome for diagnostic and therapeutic purposes, there remains a need to functionally annotate cancer-associated miRNAs and lncRNAs. Our findings help to identify the molecular roles of epigenetic processes that may contribute to cancer pathology.

Limitations of the current study include the low absolute numbers of patients who had some cancer types (lymphoma, melanoma, uterine sarcoma, and glioblastoma multiforme), which restricted our ability to perform molecular analyses for these tumors. In addition, the socioeconomic status of patients in the TCGA data are unavailable, so we were unable to adjust for this variable when comparing racial differences according to tumor biology. Also, the molecular data in TCGA lacked detailed clinical information sufficient to provide adjusted outcome measures. We consistently found higher mortality rates associated with AA race in several cancer types across 2 large cancer registries, SEER and NCDB. However, we are not able to validate data from either cohort, which is an inherent limitation of these data sources. Moreover, both data sets lack or have only incomplete treatment data, such as chemotherapy, surgical intervention, and radiation therapy. Without these important variables, we are unable to control for the biological effects of treatment on the epigenome. Finally, although we include proxies for socioeconomic status in our population-based analysis, we are unable to assess systematic differences between races. For example, patients treated in the public hospital setting are found to have a longer interval from diagnosis to surgery and fewer preoperative visits. ${ }^{49} \mathrm{We}$ also did not analyze trends in survival among other minority groups, including Asian, Native American, and 
Hispanic patients with cancer, some of whom may also have survival disadvantages. ${ }^{50}$

Hispanic patients make up a fast-growing minority group in the United States; however, the heterogeneity within this group makes molecular analysis particularly challenging. This highlights the need for additional efforts in identifying larger samples of underrepresented patients with comprehensive clinical and genomic profiles to better understand disparities in cancer survival across other minority groups.

In summary, we identified epigenetic modifications unique to AA patients with cancer that may have clinical importance because of their effects on tumor aggressiveness, response to therapy, and overall survival.

\section{FUNDING SUPPORT}

Olivia D. Lara is supported by a National Institutes of Health institutional training grant (5T32CA009599). This work was supported in part by other National Institutes of Health grants (P30CA016672, CA213759, P50CA217685, P50CA098258, and R35CA209904), the Blanton-Davis Ovarian Cancer Research Program, the American Cancer Society Research Professor Award, and the Frank T. McGraw Memorial Chair in Cancer Research (to Anil K. Sood).

\section{CONFLICT OF INTEREST DISCLOSURES}

Lin Zhang reports a research grant from Celgene outside the submitted work. Anil K. Sood reports personal fees from KIYATEC and Merck \& Company, Inc, owns stock in Bio-Path Holdings, and research funding from M-Trap outside the submitted work. The remaining authors made no disclosures.

\section{AUTHOR CONTRIBUTIONS}

Olivia D. Lara, Ying Wang, J. Alejandro Rauh-Hain, and Anil K. Sood contributed to the study design, acquired and analyzed the data, generated figures, and wrote the article. Amma Asare analyzed the data and generated the figures. Wei Hu provided intellectual input. Lin Zhang provided intellectual input and established The Cancer Genome Ancestry Atlas. Tao Xu, Hua-Sheng Chiu, Yuexin Liu, Shitanshu Uppal, and Pavel Sumazin contributed to data acquisition and statistical analysis. All authors contributed to the interpretation of data, vouched for the data analysis, contributed to the writing of the article, and agreed to the publication of this study.

\section{REFERENCES}

1. Allard JE, Maxwell GL. Race disparities between black and white women in the incidence, treatment, and prognosis of endometrial cancer. Cancer Control. 2009;16:53-56.

2. Bach PB, Schrag D, Brawley OW, Galaznik A, Yakren S, Begg CB. Survival of blacks and whites after a cancer diagnosis. JAMA. 2002;287:2106-2113.

3. Howard J, Hankey BF, Greenberg RS, et al. A collaborative study of differences in the survival rates of black patients and white patients with cancer. Cancer. 1992;69:2349-2360.

4. DeSantis CE, Miller KD, Goding Sauer A, Jemal A, Siegel RL. Cancer statistics for African Americans, 2019. CA Cancer J Clin. 2019;69:211-233.

5. Newman LA. Disparities in breast cancer and African ancestry: a global perspective. Breast J. 2015;21:133-139.

6. Shavers VL, Brown ML. Racial and ethnic disparities in the receipt of cancer treatment. J Natl Cancer Inst. 2002;94:334-357.
7. Ward E, Jemal A, Cokkinides V, et al. Cancer disparities by race/ethnicity and socioeconomic status. CA Cancer J Clin. 2004;54:78-93.

8. Williams DR, Mohammed SA, Shields AE. Understanding and effectively addressing breast cancer in African American women: unpacking the social context. Cancer. 2016;122:2138-2149.

9. Newman LA, Stark A, Chitale D, et al. Association between benign breast disease in African American and white American women and subsequent triple-negative breast cancer. JAMA Oncol. 2017;3:1102-1106.

10. Lindner R, Sullivan C, Offor O, et al. Molecular phenotypes in triple negative breast cancer from African American patients suggest targets for therapy. PLoS One. 2013;8:e71915.

11. Yuan J, Hu Z, Mahal BA, et al. Integrated analysis of genetic ancestry and genomic alterations across cancers. Cancer Cell. 2018;34:549-560. e549.

12. Ademuyiwa FO, Tao Y, Luo J, Weilbaecher K, Ma CX. Differences in the mutational landscape of triple-negative breast cancer in African Americans and Caucasians. Breast Cancer Res Treat. 2017;161:491-499.

13. Huang FW, Mosquera JM, Garofalo A, et al. Exome sequencing of African-American prostate cancer reveals loss-of-function ERF mutations. Cancer Discov. 2017;7:973-983.

14. Wang H, Schmit SL, Haiman CA, et al. Novel colon cancer susceptibility variants identified from a genome-wide association study in African Americans. Int J Cancer. 2017;140:2728-2733.

15. Feinberg AP. The key role of epigenetics in human disease prevention and mitigation. N Engl J Med. 2018;378:1323-1334.

16. Teschendorff AE, West J, Beck S. Age-associated epigenetic drift: implications, and a case of epigenetic thrift? Hum Mol Genet. 2013;22(R1):R7-R15.

17. Ahmad A, Azim S, Zubair H, et al. Epigenetic basis of cancer health disparities: Looking beyond genetic differences. Biochim Biophys Acta Rev Cancer. 2017;1868:16-28.

18. Brewer LC, Redmond N, Slusser JP, et al. Stress and achievement of cardiovascular health metrics: the American Heart Association Life's Simple 7 in blacks of the Jackson Heart Study. J Am Heart Assoc. 2018;7:e008855.

19. Grossman RL, Heath AP, Ferretti V, et al. Toward a shared vision for cancer genomic data. N Engl J Med. 2016;375:1109-1112.

20. Pounds S, Morris SW. Estimating the occurrence of false positives and false negatives in microarray studies by approximating and partitioning the empirical distribution of p-values. Bioinformatics. 2003; 19:1236-1242.

21. Li J, Han L, Roebuck P, et al. TANRIC: an interactive open platform to explore the function of lncRNAs in cancer. Cancer Res. 2015;75:3728-3737.

22. Chiu H-S, Somvanshi S, Patel E, et al. Pan-cancer analysis of IncRNA regulation supports their targeting of cancer genes in each tumor context. Cell Rep. 2018;23:297-312.

23. Subramanian A, Tamayo P, Mootha VK, et al. Gene set enrichment analysis: a knowledge-based approach for interpreting genome-wide expression profiles. Proc Natl Acad Sci U S A. 2005;102:15545-15550.

24. Geretto M, Pulliero A, Rosano C, Zhabayeva D, Bersimbaev R, Izzotti A. Resistance to cancer chemotherapeutic drugs is determined by pivotal microRNA regulators. Am J Cancer Res. 2017;7:1350-1371.

25. Jiang H, Yu WW, Wang LL, Peng Y. miR-130a acts as a potential diagnostic biomarker and promotes gastric cancer migration, invasion and proliferation by targeting RUNX3. Oncol Rep. 2015;34:1153-1161.

26. Zhang HD, Jiang LH, Sun DW, Li J, Ji ZL. The role of miR-130a in cancer. Breast Cancer. 2017;24:521-527.

27. Sueta A, Yamamoto Y, Tomiguchi M, Takeshita T, Yamamoto-Ibusuki M, Iwase H. Differential expression of exosomal miRNAs between breast cancer patients with and without recurrence. Oncotarget. 2017;8:69934-69944.

28. Ma W, Ma CN, Zhou NN, Li XD, Zhang YJ. Up-regulation of miR328-3p sensitizes non-small cell lung cancer to radiotherapy. Sci Rep. 2016;6:31651.

29. Feng Y, Bai F, You Y, et al. Dysregulated microRNA expression profiles in gastric cancer cells with high peritoneal metastatic potential. Exp Ther Med. 2018;16:4602-4608.

30. Shi Q, Zhou Z, Ye N, Chen Q, Zheng X, Fang M. MiR-181a inhibits non-small cell lung cancer cell proliferation by targeting CDK1. Cancer Biomark. 2017;20:539-546. 
31. Meijer LL, Garajova I, Caparello C, et al. Plasma miR-181a-5p downregulation predicts response and improved survival after FOLFIRINOX in pancreatic ductal adenocarcinoma. Ann Surg. Published online December 9, 2018. doi:10.1097/SLA.0000000000003084

32. Pignot G, Cizeron-Clairac G, Vacher S, et al. microRNA expression profile in a large series of bladder tumors: identification of a 3-miRNA signature associated with aggressiveness of muscle-invasive bladder cancer. Int J Cancer. 2013;132:2479-2491.

33. Lin C, Li Z, Chen P, et al. Oncogene miR-154-5p regulates cellular function and acts as a molecular marker with poor prognosis in renal cell carcinoma. Life Sci. 2018;209:481-489.

34. Aqeilan RI, Calin GA, Croce CM. miR-15a and miR-16-1 in cancer: discovery, function and future perspectives. Cell Death Differ. 2010;17:215-220.

35. Zhu Y, Gu J, Li Y, et al. MiR-17-5p enhances pancreatic cancer proliferation by altering cell cycle profiles via disruption of RBL2/E2F4repressing complexes. Cancer Lett. 2018;412:59-68.

36. Egawa $\mathrm{H}$, Jingushi $\mathrm{K}$, Hirono $\mathrm{T}$, et al. The miR-130 family promotes cell migration and invasion in bladder cancer through FAK and Akt phosphorylation by regulating PTEN. Sci Rep. 2016;6:20574.

37. Duan J, Zhang H, Qu Y, et al. Onco-miR-130 promotes cell proliferation and migration by targeting TGFbetaR2 in gastric cancer. Oncotarget. 2016;7:44522-44533.

38. He DX, Ma X. Transient receptor potential channel C5 in cancer chemoresistance. Acta Pharmacol Sin. 2016;37:19-24.

39. Ma X, Chen Z, Hua D, et al. Essential role for TrpC5-containing extracellular vesicles in breast cancer with chemotherapeutic resistance. Proc Natl Acad Sci U S A. 2014;111:6389-6394.

40. Filipska M, Skrzypski M, Czetyrbok K, et al. MiR-192 and miR-662 enhance chemoresistance and invasiveness of squamous cell lung carcinoma. Lung Cancer. 2018;118:111-118.
41. Qian J, Ding F, Luo A, Liu Z, Cui Z. Overexpression of S100A14 in human serous ovarian carcinoma. Oncol Lett. 2016;11:1113-1119.

42. Loomis D, Guyton KZ, Grosse Y, et al. Carcinogenicity of drinking coffee, mate, and very hot beverages. Lancet Oncol. 2016;17:877-878.

43. Sturtz LA, Melley J, Mamula K, Shriver CD, Ellsworth RE. Outcome disparities in African American women with triple negative breast cancer: a comparison of epidemiological and molecular factors between African American and Caucasian women with triple negative breast cancer. BMC Cancer. 2014;14:62.

44. Cote ML, Ruterbusch JJ, Olson SH, Lu K, Ali-Fehmi R. The growing burden of endometrial cancer: a major racial disparity affecting black women. Cancer Epidemiol Biomarkers Prev. 2015;24:1407-1415.

45. Deshmukh SK, Srivastava SK, Tyagi N, et al. Emerging evidence for the role of differential tumor microenvironment in breast cancer racial disparity: a closer look at the surroundings. Carcinogenesis. 2017;38:757-765.

46. Deshmukh SK, Srivastava SK, Bhardwaj A, et al. Resistin and interleukin6 exhibit racially-disparate expression in breast cancer patients, display molecular association and promote growth and aggressiveness of tumor cells through STAT3 activation. Oncotarget. 2015;6:11231-11241.

47. Martin DN, Boersma BJ, Yi M, et al. Differences in the tumor microenvironment between African-American and European-American breast cancer patients. PLoS One. 2009;4:e4531.

48. Spratt DE, Chan T, Waldron L, et al. Racial/ethnic disparities in genomic sequencing. JAMA Oncol. 2016;2:1070-1074.

49. Frey MK, Moss HA, Musa F, et al. Preoperative experience for public hospital patients with gynecologic cancer: do structural barriers widen the gap? Cancer. 2016;122:859-867.

50. Hall JE, Moonesinghe R, Bouye K, Penman-Aguilar A. Racial/ethnic disparities in mortality: contributions and variations by rurality in the United States, 2012-2015. Int J Environ Res Public Health. 2019;16:436. 